\title{
3D Printing of $\mathrm{BaTiO}_{3}$ Piezoelectric Ceramics for a Focused Ultrasonic Array
}

\author{
Jian Cheng, Yan Chen *, Jun-Wei Wu, Xuan-Rong Ji and Shang-Hua Wu
}

School of Electromechanical Engineering, Guangdong University of Technology, Guangzhou 510006, China; jiancheng0372@foxmail.com (J.C.); wujunwei@cndoppler.cn (J.-W.W.); xr.ji@gdut.edu.cn (X.-R.J.); swu@gdut.edu.cn (S.-H.W.)

* Correspondence: yanchen@gdut.edu.cn

Received: 23 August 2019; Accepted: 18 September 2019; Published: 20 September 2019

\begin{abstract}
BaTiO}_{3}$ (BTO) ceramics were fabricated based on stereolithography technology. The microstructures and electric properties of the BTO ceramics were studied. X-ray patterns of sintered BTO ceramics indicated that the tetragonal phase had formed, and the grain size increased clearly as BTO weight percentage increased. Moreover, the BTO ceramics exhibited good electric properties, with a piezoelectric constant $d_{33}$ of $166 \mathrm{pC} / \mathrm{N}$ at $80 \%$ BTO weight percentage. To evaluate the properties of 3D printed BTO ceramics, a $1.4 \mathrm{MHz}$ focused ultrasonic array was fabricated and characterized. The $-6 \mathrm{~dB}$ bandwidth of the array was $40 \%$, and the insertion loss at the center frequency was $50 \mathrm{~dB}$. The results show that the printed BTO ceramics array have good potential to be used in ultrasonic transducers for various applications.
\end{abstract}

Keywords: stereolithography; BTO; piezoelectric ceramic; ultrasonic transducer

\section{Introduction}

Transducer elements play a critical role on transducer performance, so proper piezoelectric material selection in ultrasonic transducer design is of utmost importance. Because the application of toxic lead-based materials, such as lead zirconate titanate (PZT) and (1-x) $\mathrm{Pb}\left(\mathrm{Mg}_{1 / 3} \mathrm{Nb}_{2 / 3}\right) \mathrm{O}_{3}-\mathrm{xPbTiO}_{3}$ (PMN-PT), causes serious environmental concerns, there is an urgent demand for developing lead-free substitutes for ultrasonic imaging applications. $\mathrm{BaTiO}_{3}(\mathrm{BTO})$, has been widely studied, due to its high dielectric constant, good piezoelectric coefficient, and favorable electromechanical coupling coefficient [1-4].

Ultrasonic transducers with special-shape elements and distribution are urgently needed for novel ultrasonic technology and developing new directions [5], such as brain stimulation and imaging [6,7]. However, piezoelectric ceramics with complex shapes and structures are extremely difficult to achieve by traditional manufacturing techniques, due to their brittleness. In addition, traditional machining processes, such as dicing or laser cutting, may cause strength damage and depolarization of the piezoelectric ceramics, which could deteriorate the performance of electronic devices. The additive manufacturing process, also known as 3D printing, is a rapid prototyping process having the advantages of unlimited design flexibility and low production consumption, which has attracted considerable attention in fabricating special-shape ceramics [8,9]. Kim et al. from the University of California fabricated piezoelectric composites by digital projection printing [2]. Cui et al. at Virginia Tech printed piezoelectric materials with designed anisotropy and directional response, based on functionalized PZT nanoparticle colloids [10].

A stereolithography apparatus (SLA) is a form of 3D printing technology which usually uses ultraviolet light to cure photosensitive resin. It doesn't require high energy laser beams to avoid the defects caused by internal stress. The ceramic-based components printed by SLA have high precision, 
as well as good surface quality [11,12]. There are some piezoelectric ceramics prepared using the SLA method, such as PZT [13-16], $\mathrm{Pb}\left(\mathrm{Mg}_{1 / 3} \mathrm{Nb}_{2 / 3}\right)-\mathrm{PbTiO}_{3}(\mathrm{PMN}-\mathrm{PT})$ [17], $\mathrm{K}_{0.48} \mathrm{Na}_{0.52} \mathrm{NbO}_{3}(\mathrm{KNN})$ [18], and BTO $[19,20]$. Our group printed PZT ceramics and fabricated a two-dimensional ultrasound array [16]. As for research about lead-free BTO printing, Jang et al. at Alfred University have prepared BTO UV-curable suspensions and characterized their rheological properties for stereolithography [19]. Chen et al. at the University of Southern California reported that $\mathrm{BaTiO}_{3}$ piezoelectric ceramics were printed for fabricating single-element focusing ultrasonic transducers and four-element annular piezoelectric arrays $[20,21]$. The weight percentages and microstructures of the printed BTO ceramics are essential for their properties' enhancement. However, research on the relationships between weight percentage, microstructure, and the electric properties of lead-free BTO ceramics are lacking. To better achieve high-quality BTO ceramics for device applications, the effect of weight percentage on microstructures, piezoelectric, ferroelectric, and dielectric properties were investigated for this paper. Finally, a focused ultrasonic array was fabricated to further evaluate the properties of BTO ceramics.

\section{Experimental}

\subsection{BTO Slurry and Green Parts}

BTO powders $(500 \mathrm{~nm})$ with different weight percentages $(70 \mathrm{wt} \%, 75 \mathrm{wt} \%, 80 \mathrm{wt} \%, 82 \mathrm{wt} \%$, $84 \mathrm{wt} \%$, and $86 \mathrm{wt} \%$ ) and photosensitive resin were mixed by ball-milling. The photosensitive resins were prepared with moderate amounts of the oligomers, monomer, and UV-initiating agent. In order to obtain a homogeneous slurry, dispersant was also added to break up the aggregate. Then, the BTO slurry was spread out on the resin container and then exposured by the UV light in the SLA equipment. Green parts, with a disk size of $\varphi 12 \times 3 \mathrm{~mm}$, were printed for property characterization. Then, the debinding processes of the green parts included two steps, which were carried out to obtain dense ceramics [11]. Finally, the green parts were sintered at $1290^{\circ} \mathrm{C}$ for $2 \mathrm{~h}$.

\subsection{BTO Ceramics Characterization}

The microstructures of the BTO ceramics were observed using scanning electron microscopy (SEM, LYRA 3 XMU, Tescan, Brno, CZ). The density of sintered samples was measured by the Archimedes method. An X-ray diffractometer (XRD, X'Pert PRO, PANalytical B.V., Almelo, Netherlands) was used to determine the crystal structure of the printed samples. The BTO ceramics with silver electrodes were poled under an electric field of $30 \mathrm{kV} / \mathrm{cm}$ for $15 \mathrm{~min}$ in $70{ }^{\circ} \mathrm{C}$ silicone oil using poling equipment (Rek RK2674A, HYJH-3YY/20 kV). The piezoelectric constant $\mathrm{d}_{33}$ was determined by a piezo- $\mathrm{d}_{33}$ meter (ZJ-3A, Institute of Acoustics, Chinese Academy of Sciences, Beijing, China). The room temperature dielectric constant and electromechanical coupling coefficients of the ceramics were measured using an impedance analyzer (Agilent 4294A, Agilent Technologies, Santa Clara, CA, USA). The temperature-dependent dielectric properties of the BTO ceramics were also characterized by an impedance analyzer (TH2816, Tonghui, Changzhou, China). The polarization-electric field (P-E) hysteresis loops were acquired using a ferroelectric tester (Multiferroics, Radiant Technology, Albuquerque, USA) at room temperature. The focused ultrasonic array was characterized via a traditional method based on pulse-echo response measurement.

\section{Results and Discussions}

\subsection{BTO Ceramics Characterization}

\subsubsection{Microstructure}

The SEM micrographs of 3D-printed BTO ceramics with different weight percentages are given in Figure 1. It can be seen that the grain morphology is inhomogeneous with grain size in the range of 10-15 $\mu \mathrm{m}$, and a large amount of pore appears at relatively low BTO weight percentage (70 $\mathrm{wt} \%$ ). With a BTO weight percentage increase, the grain size progressively increases, and the pore decreases 
and almost disappears when the amount of BTO exceeds $80 \%$. The results show that the microstructures of the ceramics are sensitive to BTO weight percentage. The density of sintered BTO ceramics is shown in Table 1. It can be seen that density increases with the increase of BTO weight percentage and relatively high density is obtained as the BTO weight percentage becomes larger than $80 \%$. It is well known that the BTO ceramics with higher BTO weight percentage have a larger density. However, as BTO weight percentage increases up to $82 \%$, a large amount of dispersant is added to adjust the slurry viscosity for better 3D printing, hence leading to a slightly reduction in density.
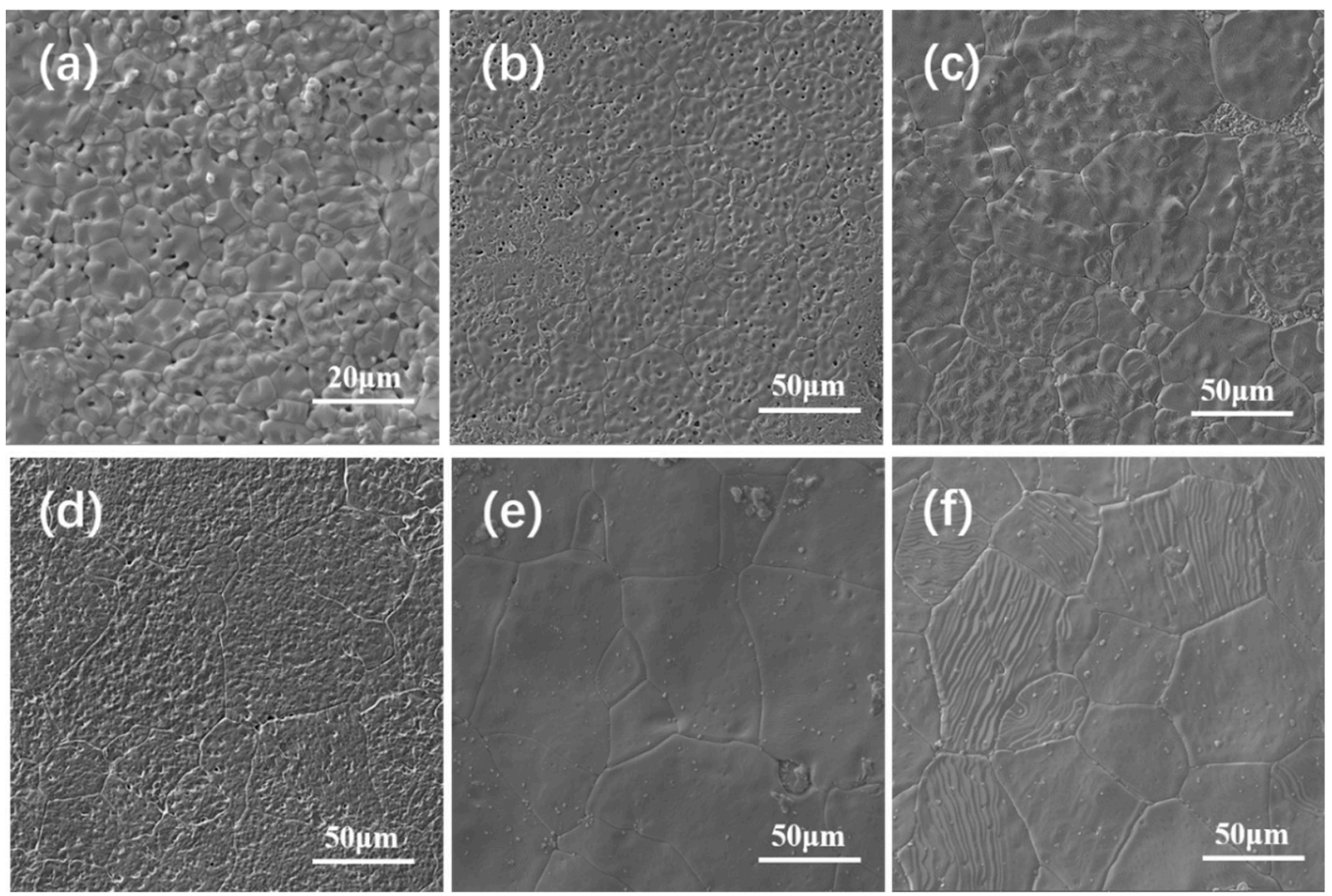

Figure 1. SEM micrographs of 3D-printed $\mathrm{BaTiO}_{3}(\mathrm{BTO})$ ceramics (a) $70 \mathrm{wt} \%$; (b) $75 \mathrm{wt} \%$; (c) $80 \mathrm{wt} \%$; (d) $82 \mathrm{wt} \%$; (e) $84 \mathrm{wt} \%$; (f) $86 \mathrm{wt} \%$.

Table 1. Density of sintered ceramics with different $\mathrm{BaTiO}_{3}(\mathrm{BTO})$ weight percentage.

\begin{tabular}{ccccccc}
\hline $\begin{array}{c}\text { BTO Weight } \\
\text { Percentage }\end{array}$ & $\mathbf{7 0} \mathbf{w t} \%$ & $\mathbf{7 5} \mathbf{w t} \%$ & $\mathbf{8 0 ~ w t} \%$ & $\mathbf{8 2} \mathbf{w t} \%$ & $\mathbf{8 4} \mathbf{w t} \%$ & $\mathbf{8 6} \mathbf{w t} \%$ \\
\hline Density $\left(\mathrm{g} / \mathrm{cm}^{\mathbf{3}}\right)$ & 5.52 & 5.57 & 5.65 & 5.62 & 5.63 & 5.68 \\
\hline
\end{tabular}

\subsubsection{XRD Patterns}

Figure 2 shows the XRD patterns of 3D-printed ceramics with different BTO weight percentages. It can be seen that all BTO ceramics show a tetragonal perovskite structure, the intensity of XRD patterns increase, and the diffraction peaks become sharp as the BTO weight percentage increases, which indicates that good crystallinity can be formed and may also be affected by the increased grain size. 


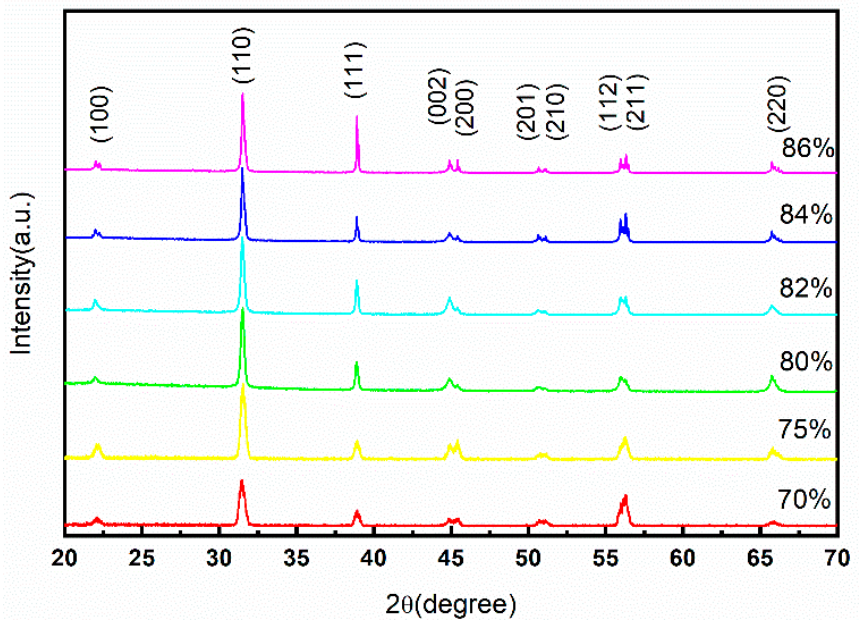

Figure 2. XRD patterns of $\mathrm{BaTiO}_{3}$ ceramics with different weight percentages.

\subsubsection{Electrical Properties}

In order to characterize the properties of the 3D-printed BTO ceramics, ceramic disks (diameter $10 \mathrm{~mm}$, thickness $\sim 0.6 \mathrm{~mm}$ ) were prepared using the above fabrication process. The electrical properties of the BTO ceramics with different weight percentages are shown in Table 2 . The piezoelectric constant $d_{33}$ increases from 96 to $166 \mathrm{pC} / \mathrm{N}$, almost reaching the maximum value, as the BTO weight percentage increases to $80 \%$, and then decreases a little. The weak properties with relatively low BTO weight percentage $(\leq 75 \%)$ may be related to the low density affected by the pores. Dielectric properties also showed the same phenomena for the samples with lower BTO weight percentages $(\leq 75 \%)$. The electromechanical coefficient $k_{t}$ is in the range of $0.173-0.416$. The enhancement of the electrical properties may be related to improved density and increased grain size as the BTO weight percentage increases. The reasons for the decrease of the piezoelectric constant after $80 \mathrm{wt} \%$ can be explained by the effects of more dispersant additions.

Table 2. The electrical properties of the 3D-printed BTO ceramics.

\begin{tabular}{cccccccc}
\hline $\begin{array}{c}\text { BTO Weight Percentage } \\
\text { Electrical Properties }\end{array}$ & $\mathbf{7 0} \mathbf{w t} \%$ & $\mathbf{7 5} \mathbf{w t} \%$ & $\mathbf{8 0} \mathbf{w t} \%$ & $\mathbf{8 2} \mathbf{w t} \%$ & $\mathbf{8 4} \mathbf{w t} \%$ & $\mathbf{8 6} \mathbf{w t} \%$ & BTO Ceramic [22] \\
\hline $\mathrm{d}_{33}(\mathrm{pC} / \mathrm{N})$ & 96 & 110 & 166 & 130 & 124 & 122 & 190 \\
$\varepsilon_{\mathrm{r}}(1 \mathrm{kHz})$ & 1829 & 2175 & 2177 & 2210 & 2251 & 2276 & 1700 \\
$\tan \delta$ & 0.094 & 0.076 & 0.036 & 0.040 & 0.036 & 0.036 & $<0.1$ \\
$\mathrm{k}_{\mathrm{t}}$ & 0.29 & 0.36 & 0.41 & 0.42 & 0.39 & 0.17 & - \\
\hline
\end{tabular}

To investigate the effects of BTO weight percentage on ferroelectric properties, the room temperature ferroelectric hysteresis loop of the printed samples, with different BTO weight percentages, was measured under an electric field of $30 \mathrm{kV} / \mathrm{cm}$ at $10 \mathrm{~Hz}$ (Figure 3a). Both the remnant polarization and coercive field increase as the BTO weight percentage increases to $80 \%$, then remains almost unchanged. The increased remnant polarization may be explained by the decrease of barrier height for the ceramics, which is affected by the increased gain size and high density. The enlarged coercive field indicates that more energy is needed for the movement and reorientation of domain walls. Moreover, for the sake of better understanding the ferroelectric properties of the $80 \%$ BTO sample, the polarization-electric field(P-E) loops under different electric fields are shown in Figure $3 \mathrm{~b}$. The P-E loops become saturated as the electric field increases and the remnant polarization and coercive fields increase to $28.5 \mu \mathrm{C} / \mathrm{cm}^{2}$ and $10 \mathrm{kV} / \mathrm{cm}$, respectively. The ferroelectric properties of the printed samples are comparable to that of BTO ceramics fabricated by using a traditional pressing method [22]. 


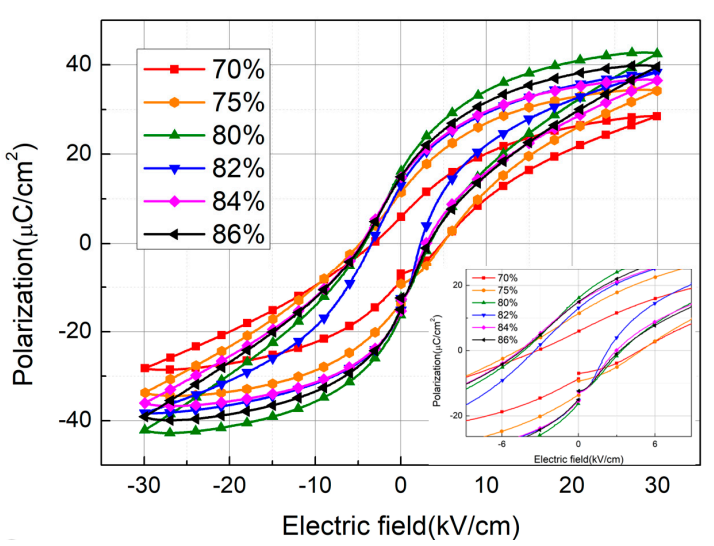

(a)

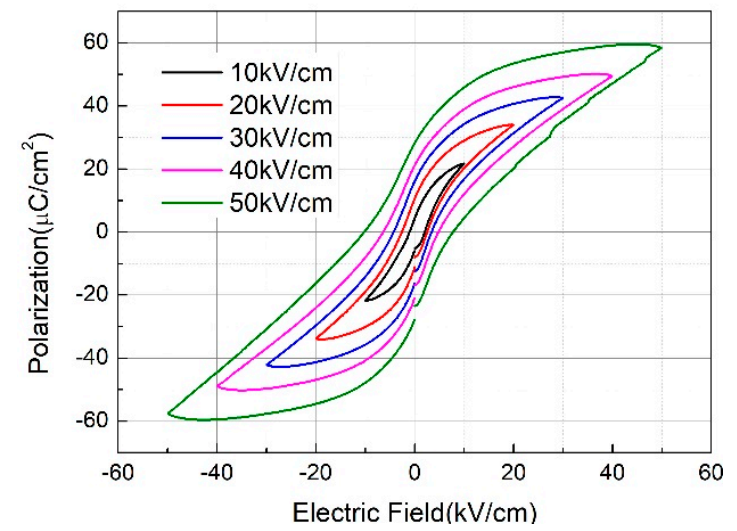

(b)

Figure 3. Polarization-electric field hysteresis loops of the printed ceramics with (a) different BTO weight percentages under $30 \mathrm{kV} / \mathrm{cm}$ (inset is the magnified one), (b) $80 \%$ BTO weight percentage under different electric fields.

The temperature dependences of the dielectric constant and dielectric loss at $1 \mathrm{kHz}$ for printed BTO ceramics are shown in Figure 4. All printed samples show only one dielectric peak at about $120^{\circ} \mathrm{C}$, which corresponds to the Curie temperature. It can clearly be seen that the Curie temperature increases slightly as BTO weight percentage increases (Figure $4 \mathrm{~b}$ ), which may also be related to the increase of grain size, contributing to growth of the domains. The dielectric loss of all samples is smaller than 0.05 from room temperature to $130{ }^{\circ} \mathrm{C}$. In summary, the $80 \%$ BTO weight percentage with relatively good properties was selected to fabricate the focused ultrasonic array.

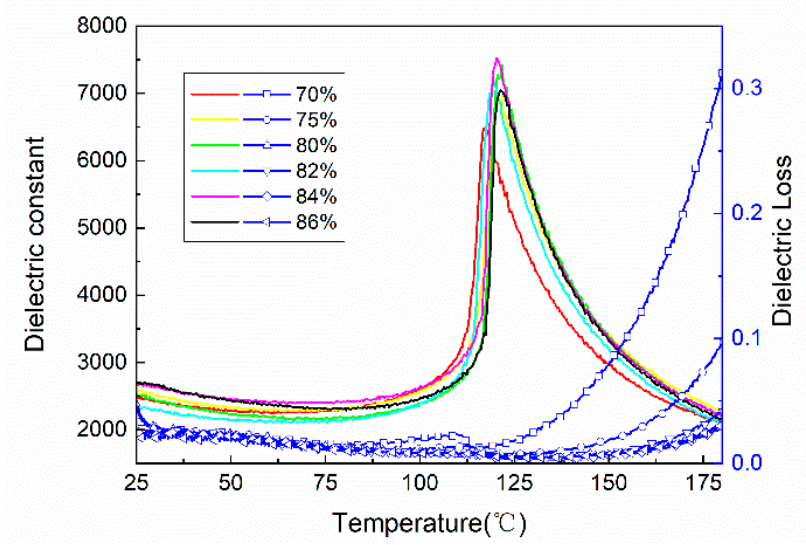

(a)

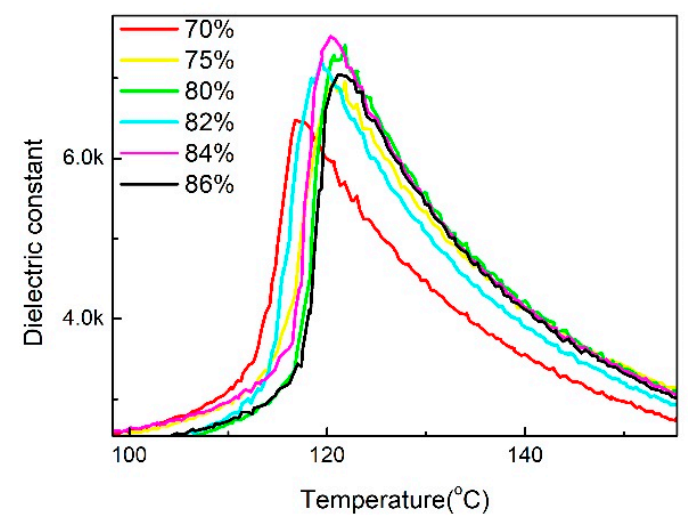

(b)

Figure 4. The temperature dependence of the dielectric constant and (a) dielectric loss, and the (b) magnified figure around Curie temperature.

\subsection{D Printing Focused Ultrasonic Transducer}

Here, a focused array was designed and fabricated with $80 \%$ BTO ceramics based on the SLA method. Figure 5 shows the photos of the 3D-printed BTO ceramic array and transducers. The ceramic array was printed with a curvature radius of $20.3 \mathrm{~mm}$ and thickness of $1.8 \mathrm{~mm}$. The pitch and kerf are $4.5 \mathrm{~mm}$ and $0.5 \mathrm{~mm}$, respectively. The prototype of the focused ultrasonic array is composed of BTO ceramics (as the active element), a backing layer, and cables. The backing layer, with a thickness of $10 \mathrm{~mm}$, is casted onto the ceramic using a low-viscosity epoxy (Epo-Tek 301, Epoxy Technology, Billerica, MA, USA) mixed with tungsten powder and microbubbles. The acoustic impedance of the 
backing layer is about 5 MRalys. The kerfs between each element are filled with epoxy to reduce crosstalk. The tested array is placed in a water tank and towards a quartz target. An ultrasound pulser/receiver (JSR Ultrasonics DPR500, Pittsford, NY, USA), as well as an oscilloscope (Keysight DSOS054A), are connected to the element for characterization. Figure 6 shows the tested pulse-echo waveform and frequency spectra of the focused array. A 1.4 MHz focused array shows a wide bandwidth of $40 \%$ at $-6 \mathrm{~dB}$, and its insertion loss is about $50 \mathrm{~dB}$, which exhibits promising performance when compared to the traditional PZT array [23]. The above results suggest that the 3D-printed BTO ceramics have good application prospects for complex-structure ultrasonic arrays.

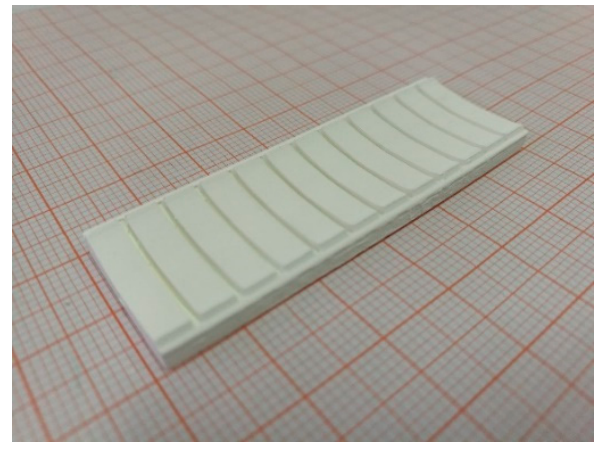

(a)

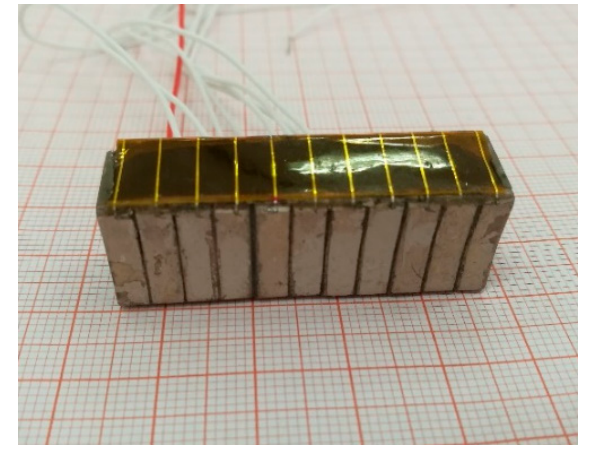

(b)

Figure 5. The photos of the 3D-printed (a) ceramic and (b) ultrasonic arrays.

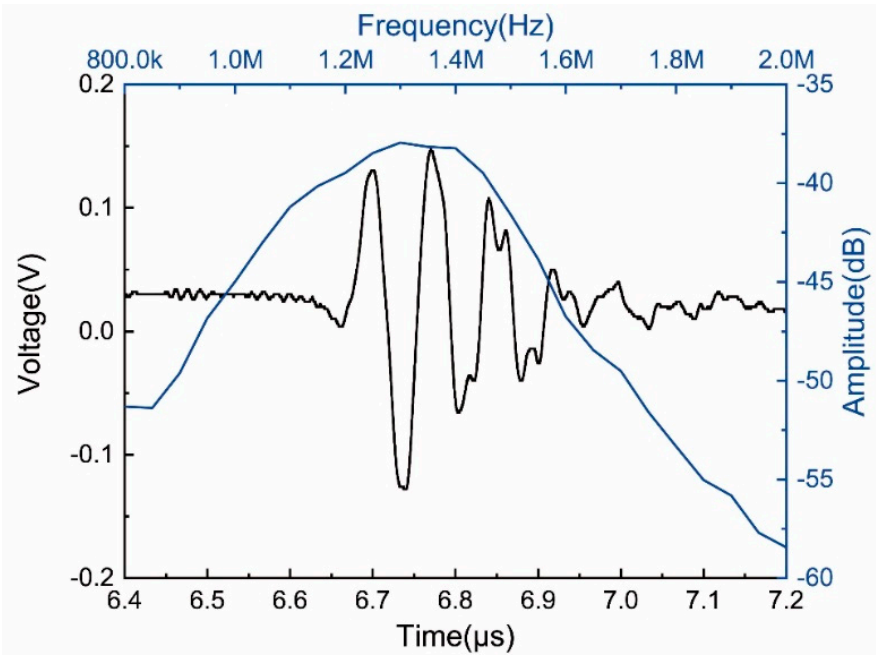

Figure 6. The pulse-echo waveform and frequency spectra of the focused array.

\section{Conclusions}

3D-printed BTO ceramics with different BTO weight percentages (70\%-86\%) were prepared using stereolithography. The piezoelectric, ferroelectric, and dielectric properties of the 3D-printed ceramics were studied. The electric properties of ceramics clearly increased and then reduced slightly at $80 \mathrm{wt} \%$ with the increase of BTO weight percentage. A focused ultrasonic array with good performance was fabricated using $80 \%$ BTO ceramics, which demonstrated that 3D-printed BTO ceramics have good application prospects for complex-shape ultrasonic arrays.

Author Contributions: J.C. and Y.C. wrote and edited the manuscript and performed the experiments. J.-W.W. performed the experiments of the focused array. X.-R.J. and S.-H.W. conceived the project and edited the manuscript. 
Funding: This work was financially supported by Guangdong Innovative and Entrepreneurial Research Team Program (Grant No. 2016ZT06G375), National Science Foundation Grants (NSFC Grant No. 11804059) and Jihua Laboratory Project (Grant No.X190061UZ190).

Conflicts of Interest: The authors declare no conflict of interest.

\section{References}

1. Gao, J.-H.; Xue, D.-Z.; Liu, W.-F.; Zhou, C.; Ren, X.-B. Recent Progress on $\mathrm{BaTiO}_{3}$-Based Piezoelectric Ceramics for Actuator Applications. Actuators 2017, 6, 24. [CrossRef]

2. Kim, K.; Zhu, W.; Qu, X.; Aaronson, C.; McCall, W.R.; Chen, S.-C.; Sirbuly, D.J. 3D Optical Printing of Piezoelectric Nanoparticle-polymer Composite Materials. ACS Nano 2014, 8, 9799-9806. [CrossRef] [PubMed]

3. Park, J.Y.; Kang, Y.Y.; Yoon, H.W.; Park, N.K.; Jo, Y.-J.; Jeong, S.-H.; Won, J.C.; Kim, Y.H. Viscoelastic Properties of a 3D-Printable High-dielectric Paste with Surface-modified BaTiO3. Compos. Sci. Technol. 2018, 159, 225-231. [CrossRef]

4. Rouffaud, R.; Granger, C.; Hladky-Hennion, A.-C.; Thi, M.P.; Levassort, F. Tonpilz Underwater Acoustic Transducer Integrating Lead-free Piezoelectric Material. Phys. Procedia 2015, 70, 997-1001. [CrossRef]

5. Matuda, M.Y.; Buiochi, F.; Adamowski, J.C. Imaging through a Convex Interface with Unknown Position and Shape using an ultrasonic linear array. AIP Conf. Proc. 2012, 1433, 177-180.

6. Landhuis, E. Ultrasound for the brain. Nature 2017, 551, 257-259. [PubMed]

7. Xia, J.; Yang, Y.; Hu, C.; Meng, R.; Jiang, Q.; Liu, R.; Yu, Y.; Sheng, Z.; Yan, F.; Zhang, L.; et al. Evaluation brain tumor in small animals using plane-wave-based power Doppler imaging. Ultrasound Med. Biol. 2019, 45, 811-822. [CrossRef] [PubMed]

8. Deckers, J.; Vleugels, J.; Kruth, J.P. Additive Manufacturing of Ceramics: A Review. J. Ceram. Sci. Tech. 2014, 5, 245-260.

9. Travitzky, N.; Bonet, A.; Dermeik, B.; Fey, T.; Filbert-Demut, I.; Schlier, L.; Schlordt, T.; Greil, P. Additive Manufacturing of Ceramic-based Materials. Adv. Eng. Mater. 2014, 16, 729-754. [CrossRef]

10. Cui, H.; Hensleigh, R.; Yao, D.; Maurya, D.; Kumar, P.; Kang, M.G.; Priya, S.; Zheng, X. Three-dimensional printing of piezoelectric materials with designed anisotropy and directional response. Nat. Mater. 2019, 18, 234-241. [CrossRef]

11. Li, K.; Zhao, Z. The Effect of the Surfactants on the Formulation of UV-curable SLA Alumina suspension. Ceram. Int. 2017, 43, 4761-4767. [CrossRef]

12. Chen, Z.; Li, Z.; Li, J.; Liu, C.; Lao, C.; Fu, Y.; Liu, C.; Li, Y.; Wang, P.; He, Y. 3D Printing of Ceramics: A Review. J. Eur. Ceram. Soc. 2019, 39, 661-687. [CrossRef]

13. Dufaud, O.; Marchal, P.; Corbel, S. Rheological Properties of PZT Suspensions for Stereolithography. J. Eur. Ceram. Soc. 2002, 22, 2081-2092. [CrossRef]

14. Singh, P.; Smith, S.; Bezdecny, M.; Cheverton, M.; Brewer, J.A.; Venkataramani, V. Additive Manufacturing of PZT-5H Piezoceramic for Ultrasound Transducers. In Proceedings of the IEEE International Ultrasonics Symposium, Orlando, FL, USA, 18-21 October 2011; pp. 1111-1114.

15. Yang, Y.; Chen, Z.-Y.; Song, X.; Zhu, B.-P.; Hsiai, T.; Wu, P.-I.; Xiong, R.; Shi, J.; Chen, Y.; Zhou, Q.-F.; et al. Three Dimensional Printing of High Dielectric Capacitor using Projection based Stereolithography Method. Nano Energy 2016, 22, 414-421. [CrossRef]

16. Chen, Y.; Bao, X.-L.; Wong, C.-M.; Cheng, J.; Wu, H.-D.; Song, H.-Z.; Ji, X.-R.; Wu, S.-H. PZT Ceramics Fabricated based on Stereolithography for An Ultrasound Transducer Array Application. Ceram. Int. 2018, 44, 22725-22730. [CrossRef]

17. Woodward, D.I.; Purssel, C.P.; Billson, D.R.; Hutchins, D.A.; Leigh, S.J. Additively-Manufactured Piezoelectric Devices. Phys. Status Solidi A Appl. Mater. Sci. 2015, 212, 2107-2113. [CrossRef]

18. Chen, W.; Wang, F.-F.; Yan, K.; Zhang, Y.-H.; Wu, D.-W. Micro-Stereolithography of KNN-based Lead-free Piezoceramics. Ceram. Int. 2019, 45, 4880-4885. [CrossRef]

19. Jang, J.H.; Wang, S.; Pilgrim, S.M.; Schulze, W.A. Preparation and Characterization of Barium Titanate Suspensions for Stereolithography. J. Am. Ceram. Soc. 2000, 83, 1804-1806. [CrossRef]

20. Chen, Z.-Y.; Song, X.; Lei, L.-W.; Chen, X.-Y.; Fei, C.-L.; Chiu, C.T.; Qian, X.-J.; Ma, T.; Yang, Y.; Shung, K.; et al. 3D Printing of Piezoelectric Element for Energy Focusing and Ultrasonic sensing. Nano Energy 2016, 27, 78-86. [CrossRef] 
21. Chen, Z.-Y.; Qian, X.-J.; Song, X.; Jiang, Q.-G.; Huang, R.-J.; Yang, Y.; Li, R.-Z.; Shung, K.; Chen, Y.; Zhou, Q.-F. Three-Dimensional Printed Piezoelectric Array for Improving Acoustic Field and Spatial Resolution in Medical Ultrasonic Imaging. Micromachines 2019, 10, 170. [CrossRef]

22. Vijatovic, M.M.; Bobic, J.D.; Stojanovic, B.D. History and Challenges of Barium Titanate: Part II. Sci. Sintering 2008, 40, 235-244. [CrossRef]

23. Chen, Y.; Zhou, D.; Lam, K.-H.; Cheung, K.-F.; Dai, J.-Y.; Chan, H.L.-W. Endoscopic Ultrasound Radial Array Transducers Fabricated with PZT Tube by a Rotate-and-dice Method. Sens. Actuators A Phys. 2013, 201, 357-362. [CrossRef]

(C) 2019 by the authors. Licensee MDPI, Basel, Switzerland. This article is an open access article distributed under the terms and conditions of the Creative Commons Attribution (CC BY) license (http://creativecommons.org/licenses/by/4.0/). 